\title{
Using process evaluation to determine effectiveness of participatory ergonomics training interventions in construction
}

\author{
Ann Marie Dale ${ }^{\mathrm{a}^{*}}$, Lisa Jaegers ${ }^{\mathrm{a}, \mathrm{b}}$, Brian Buchholz ${ }^{\mathrm{c}}$, Laurie Welch ${ }^{\mathrm{d}}$, and Bradley A. Evanoff $\mathrm{f}^{\mathrm{a}}$, \\ ${ }^{a}$ Department of Medicine, Washington University, Campus Box 8005, 660 S. Euclid, St. Louis, MO \\ 63110, USA \\ ${ }^{b .}$ School of Public Health, Saint Louis University 3545 Lafayette Avenue, St. Louis, MO 63104, USA \\ c. University of Massachusetts Lowell, 1 University Ave, Lowell, MA 01854, USA \\ d. The Center for Construction Research and Training CPWR, 8484 Georgia Ave, Silver Spring, MD \\ 20910, USA
}

\begin{abstract}
The construction industry continues to experience high rates of musculoskeletal injuries despite the widespread promotion of ergonomic solutions. Participatory ergonomics (PE) has been suggested as one approach to engage workers and employers for reducing physical exposures from work tasks but a systematic review of participatory ergonomics programs showed inconclusive results.. A process evaluation is used to monitor and document the implementation of a program and can aid in understanding the relationship between the program elements and the program outcomes. The purpose of this project is to describe a proposed process evaluation for use in a participatory ergonomic training program in construction workers and to evaluate its utility in a demonstration project among floor layers.
\end{abstract}

Keywords: musculoskeletal disorders, implementation strategy, work measurement

\section{Introduction}

Participatory ergonomics training interventions have produced inconsistent reductions in physical exposures and worker symptoms when used as the primary method for addressing musculoskeletal disorders in several work populations [1]. Reviews of past studies involving participatory ergonomic interventions reported inadequate description of the intervention [2] and implementation strategies [3], and limited use of process evaluation [4-6] to measure the program effectiveness. There are few details about workers' response to training, ability to implement intervention, and sustainability of new work methods over time. Lack of these data limits interpretation of a change in physical exposures or health outcomes: it is often unclear if a "negative" result is due to lack of effectiveness of the participatory ergonomics program, or lack of effective delivery of the program.

Process evaluation provides the framework to guide program planning, ensure delivery, and quantify the degree that the program was delivered. The purpose of this project is to describe a proposed process evaluation for use in a participatory ergonomic training program in construction workers and to evaluate its utility in a demonstration project among floor layers.

\section{Method}

A recently funded 5-year project is designed to determine whether participatory ergonomic interventions reduce physical exposures and improve health outcomes in three construction trades: floor layers, carpenters, and sheet metal workers. Baseline selfreported health status and assessment of physical exposures in work tasks will be compared to the same measures after delivery of the program. The participatory ergonomics intervention will be con-

${ }^{*}$ Corresponding author. Tel. No.: 011+1+314+454+8470 Email: adale@dom.wustl.edu 
ducted in small groups of workers over a period of up to 6 months time. As part of this larger project, a process evaluation has been proposed to evaluate delivery of the program. The participatory ergonomics training program and proposed process evaluation were delivered to a group of floor layers and will be the focus of this presentation. The training program contains two phases: general training and problemsolving group facilitation. Phase I consists of education in ergonomic principles and problem-solution development in a group format. Training was planned for completion within 2 hours during two sessions. Measures recorded include attendance, achievement of the training objectives, and posttraining knowledge scores. Phase II includes facilitation of the group problem-solving process to review the weekly status of using suggested solutions for recognized problems. Process evaluation measures include one-on-one researcher-worker interactions to discuss identified problems within work tasks and proposed solutions, frequency of worker-researcher interactions, responses on survey to assess worker attitude, perceived control, and willingness to change work behaviors. Summary of interactions record the individual and group's compliance to solutions, and describe barriers to implementation of solutions. Individual process measures include researcher's observed time of compliance with solutions for each worker, worker reported compliance, reasons for non-compliance, and suggested modifications to solutions.

Physical exposures of the targeted high risk tasks are measured throughout the period of time for delivery of the program to determine the proportion of task time the solution was utilized and the intensity of exposures in tasks. The outcomes of the process evaluation will show the number of problems identified and successfully addressed, list of barriers to implementation, and proportion of task time addressed by the solution and reduction in intensity level of physical exposures in targeted tasks. Worker surveys will show health status at the end of the program.

\section{Results}

Preliminary results on a group of sixteen floor layers are under analysis following participation for three months of an ongoing participatory ergonomics training program. The workers were male and Caucasians with a mean age of 29 years and mean years working in the floor laying trade of eight years. At baseline, self-reported average daily time spent with a back bent posture was 7.4 hours (SD 1.2), kneeling posture was 7.9 hours (SD 0.7), and gripping tools was 6.7 hours (SD 1.4). Sixty-nine percent of the workers reported some pain or discomfort in the last 4 weeks. Almost all workers $(93 \%)$ felt pain or discomfort in the last 6 months with 53\% missing days at work or seeking physician care due to the pain or discomfort.

All workers attended the complete or an abbreviated version of Phase I training program with objectives met during the training. Average post training knowledge scores were $86 \%$ correct with review of objectives for missed items following training. On follow-up survey, all attendees reported the training program provided useful information although $82 \%$ preferred learning through one-on-one interactions or the Phase II Problem-solution identification module. After three months of the program, there were 90 worker interactions from one-on-one and weekly meetings with more than 92 solutions identified for problematic floor laying tasks. For example, low back discomfort was identified from back bent posture to spray sealant from a can on the floor. The worker-identified solution was to use an extended handle for the spray can. In a second example, wrist discomfort was recognized caused by hand pulling glued down carpet to remove it from the floor. The worker and researcher recognized that use of an electric carpet puller would reduce the exposure although the worker must choose to use the device if it was made available.

Additional review of results will examine worker compliance with solutions and reasons for barriers to compliance. Assessment of worker's willingness to change will be compared to their ability to identify solutions and compliance with recognized solutions. Follow-up health status and report of physical exposures will be examined.

\section{Discussion}

Process measures are a critical but often overlooked aspect of intervention evaluation, important for both internal and external validity. To address limitations described in previous studies, we present process measures intended for evaluation of an ongoing participatory ergonomics intervention among construction workers. Interpreting and evaluating the results of complex interventions requires detailed 
data on the delivery of the intervention. Process evaluation can provide researchers and ergonomists a greater understanding of how well intervention programs reduce physical exposures.

\section{Acknowledgements}

This study is supported by the Centers for Disease Control and Prevention, National Institute of Occupational Safety and Health and the Center for Construction Research and Training, Grant \# NIOSH U60 $\mathrm{OH} 009762$.

\section{References}

[1] D. van Eerd, D. Cole, E. Irvin, Q. Mahood, K. Keown, N. Theberge, J. Village, M. St. Vincent, and K. Cullen, Process and implementation of participatory ergonomic interventions: a systematic review, Ergonomics (2010), 1153-1166.

[2] I. Rivilis, D. Van Eerd, K. Cullen, D.C. Cole, E. Irvin, J. Tyson and Q. Mahood, Effectiveness of participatory ergonomic interventions on health outcomes: a systematic review, Appl Ergon (2008), 342-58.
[3] H.F. van der Molen, J.K. Sluiter, C.T. Hulshof, P. Vink, and M.H. Frings-Dresen, Effectiveness of measures and implementation strategies in reducing physical work demands due to manual handling at work, Scand J Work Environ Health (2005), 75-87.

[4] M. Driessen, K. Proper, J. Anema, P. Bongers, and A. van der Beek, Process evaluation of participatory ergonomics program to prevent low back pain and neck pain among workers, Implementation Science (2010), 1-11.

[5] M. St-Vincent, M. Bellemare, G. Toulouse, and C. Tellier, Participatory ergonomic processes to reduce musculoskeletal disorders: Summary of a Québec experience, Work (2006), 123-135.

[6] Y. Roquelaure, Workplace intervention and musculoskeletal disorders: the need to develop research on implementation strategy, Occup Environ Med (2008), 4-5. 